\title{
Use of SLODAR for daytime turbulence profiling
}

\author{
Elizabeth Carlisle, Dirk Schmidt, Jose Marino, Andrés Guesalaga
}

October 15, 2017

\begin{abstract}
We adapt the Slope Detection and Ranging (SLODAR) technique, a method for characterizing the vertical turbulence profile of the atmosphere, for use in solar telescopes with a Shack-Hartmann wavefront sensor. Our algorithm is based on the SLODAR system modified to use multiple wavefront sensing directions for the Gemini South Multi-Conjugate Adaptive Optics System. We present results from a study to determine its accuracy when extended objects (solar granulation) are used for wavefront sensing. We use computer simulations to explore the performance and reliability of the algorithm. One major focus is to understand its reduced sensitivity with height, which is particularly important in the solar case. The aim of this study is to validate the SLODAR method for solar applications, improve its performance, and determine the best design to deploy to Clear, the MCAO system at the Big Bear Solar Observatory (BBSO); and to the Daniel K. Inouye Solar Telescope (DKIST).
\end{abstract}

\section{INTRODUCTION}

An understanding of the turbulence profile of the atmosphere $\left(C_{n}^{2}\right)$ can be used to optimize adaptive optics (AO) systems. Traditional AO operates under the assumption that most of the contribution to atmospheric turbulence comes from the ground layer. Modern multi-conjugate adaptive optics (MCAO) systems, however, are capable of correcting multiple layers of turbulence by conjugating deformable mirrors to the correct heights, thus increasing the correction over a wider field of view. Knowledge of the turbulence profile allows us optimize the positions of the mirrors. Therefore, it is extremely useful to have a reliable method for measuring $\left(C_{n}^{2}\right)$.

The Slope Detection and Ranging (SLODAR) algorithm, developed in $2002,{ }^{2}$ is a reliable method used to determine the height of turbulence layers based on taking a covariance of the information given from a WFS in multiple sensing directions. Our goal is to provide a working implementation of the SLODAR method for Clear, the MCAO system at the Goode Solar Telescope (GST, formerly known as NST). The GST is currently the largest clear aperture solar telescope at $1.6 \mathrm{~m}$, and is located at Big Bear Solar Observatory.

However, SLODAR has not previously been tested in a solar telescope. Here, we present results of a preliminary study to determine the performance of SLODAR with a solar application. Our algorithm is based on an adaptation of SLODAR developed at Gemini for use with multiple guide stars. ${ }^{1}$ We wish to understand the limits of this system and determine how to optimize parameters in order to improve its overall performance. As there is currently no working SLODAR system at a solar telescope, we must rely on computer simulations to predict how the algorithm will perform when implemented with a real solar AO system. 


\section{THE SLODAR METHOD}

The SLODAR algorithm uses triangulation to determine the height of turbulence layers in the atmosphere. In the example diagram in Figure 1, when the turbulence layer is at the height of the line shown, the shifts of adjacent subapertures will have a high covariance, because the light arriving at both subapertures will have passed through the same section of the layer. As the turbulence layer moves higher, the distance between subapertures with a maximum shift covariance increases. The separation between subapertures with maximum covariance is $\frac{H \theta}{\omega}$ where $H$ is the height of the layer, $\theta$ is the angular separation between guide stars, and $\omega$ is the subaperture width.

To use SLODAR, we start by making a set of reference functions that will be used to fit data to, which is generally a set of matrices that describe the covariance between shift data from the WFS with the corresponding data for a different sensing direction. These covariance matrices can be made either numerically in a Monte Carlo simulation, or calculated analytically. To produce them numerically, we use use simulations to calculate the WFS shifts from many different random phase screens (10,000 phase screens tends to give a good result). Each matrix is then calculated by the covariance of shifts in each subaperture with every other subaperture. A covariance matrix for a height of $6 \mathrm{~km}$ is shown in Figure 2. Each box in the image is the covariance of all subapertures from one direction with all subapertures in a different direction, and there is one box for each of the 9 unique baselines. Using simulated data, we produce a matrix such as this for every $500 \mathrm{~m}$ from 0 to $18 \mathrm{~km}$.

It is also possible to calculate the required covariances analytically, using known atmospheric statistics. For the covariance between two subapertures we use

$$
\iiint \int\left\langle\Phi\left(x_{1}, y_{1}\right) \Phi\left(x_{2}, y_{2}\right)\right\rangle F\left(x_{1}, y_{1}\right) F\left(x_{2}, y_{2}\right) W\left(x_{1}, y_{1}\right) W\left(x_{2}, y_{2}\right) \mathrm{d} x_{1} \mathrm{~d} y_{1} \mathrm{~d} x_{2} \mathrm{~d} y_{2}
$$

where $x_{1}$ and $y_{1}$, and $x_{2}$ and $y_{2}$ are the $\mathrm{x}$ - and $\mathrm{y}$ - distances, in units of subapertures, from the centers of the first and second subaperture, respectively. $F$ is the tilt function, and $W$ is a function that returns 1 if the given points are inside the pupil, and 0 if outside. The covariance of the phase, $\left\langle\Phi\left(x_{1}, y_{1}\right) \Phi\left(x_{2}, y_{2}\right)\right\rangle$, can be calculated using

$$
\begin{aligned}
\left\langle\Phi\left(x_{1}, y_{1}\right) \Phi\left(x_{2}, y_{2}\right)\right\rangle= & -\frac{1}{2} D\left(u_{x}+x_{2}-x_{1}, u_{y}+y_{2}-y_{1}\right) \\
& +\frac{1}{2} \iint W\left(x_{1}, y_{1}\right) D\left(u_{x}+x_{2}-x_{1}, u_{y}+y_{2}-y_{1}\right) \mathrm{d} x_{1} \mathrm{~d} y_{1} \\
& +\frac{1}{2} \iint W\left(x_{2}, y_{2}\right) D\left(u_{x}+x_{2}-x_{1}, u_{y}+y_{2}-y_{1}\right) \mathrm{d} x_{2} \mathrm{~d} y_{2} \\
& -\frac{1}{2} \iiint \int W\left(x_{1}, y_{1}\right) W\left(x_{2}, y_{2}\right) D\left(u_{x}+x_{2}-x_{1}, u_{y}+y_{2}-y_{1}\right) \mathrm{d} x_{1} \mathrm{~d} y_{1} \mathrm{~d} x_{2} \mathrm{~d} y_{2}
\end{aligned}
$$

where $D$ is the structure function of the phase aberrations. The separation distances in the $\mathrm{x}$ - and y- directions, $u_{x}$ and $u_{y}$, are measured in units of subapertures, and are offset by $\Delta_{x}$ and $\Delta_{y}$, the location of the peak covariance, such that $u_{x}=i^{\prime}-i+\Delta_{x}$ and $u_{y}=j^{\prime}-j+\Delta_{y} . \Delta$ is found using $\frac{H \theta}{w}$, where $H$ is the height of the turbulence layer, $\theta$ is the $\mathrm{x}$ - or $\mathrm{y}$-component of the angle between guide stars, and $w$ is the subaperture width. ${ }^{3}$ 
A covariance matrix is then made using the real data to be tested, and it is fit to the reference functions to obtain a turbulence profile. In Figure 3, a covariance matrix produced using three simulated layers of atmospheric turbulence (at $0 \mathrm{~km}, 3 \mathrm{~km}$, and $6 \mathrm{~km}$ ) is shown. This matrix should be equal to adding together the reference functions for $0 \mathrm{~km}, 3 \mathrm{~km}$, and $6 \mathrm{~km}$.

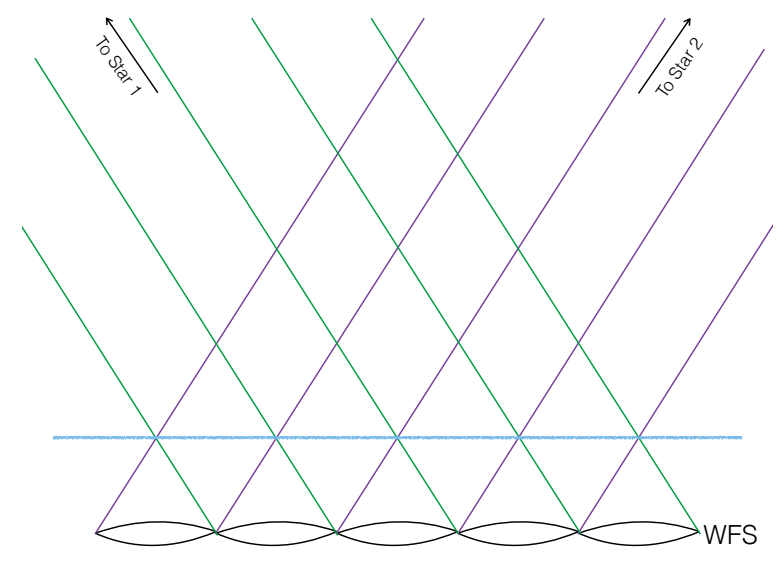

Figure 1. A diagram of SLODAR's triangulation technique. If the blue line is a turbulence layer, the shifts of a subaperture for star 2 will have a high covariance with the shifts for star 1 of the subaperture immediately to the right.

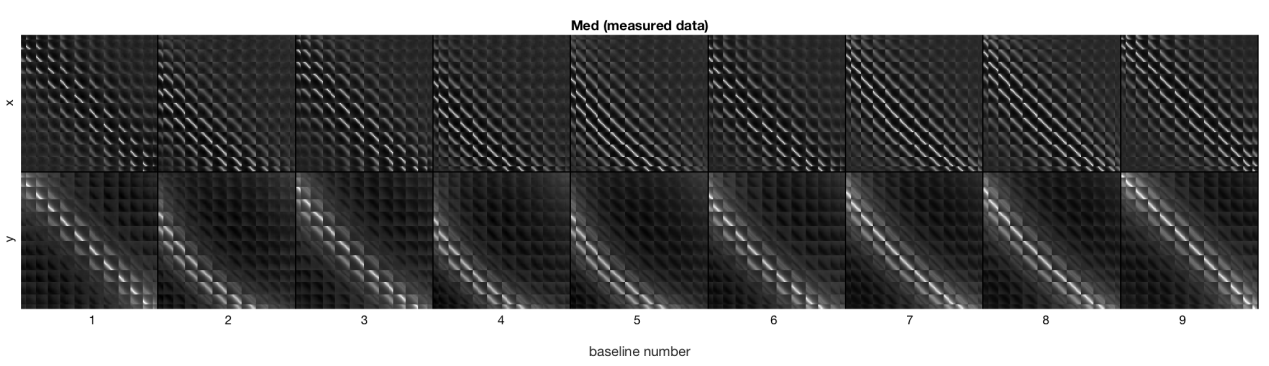

Figure 2. A covariance matrix corresponding to a turbulence layer at $6 \mathrm{~km}$. Each box represents a different baseline. 


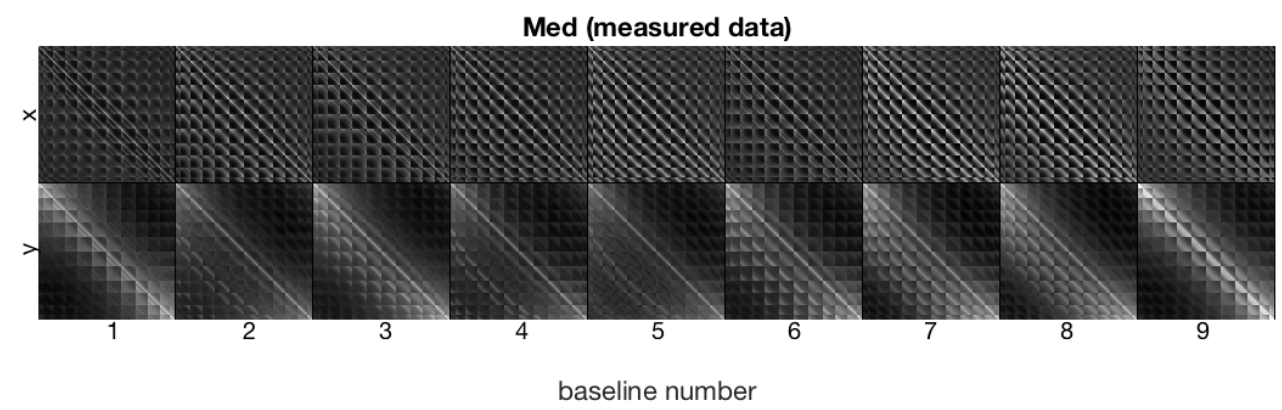

Figure 3. A covariance matrix corresponding to turbulence layers at 0,3 , and $6 \mathrm{~km}$.

\section{THE TESTING ENVIRONMENT}

\subsection{Adaptive optics simulation}

The WFS data used in our analysis was generated by a solar AO and MCAO simulation software package called Blur. Using simulated data provides a flexible controlled environment where we can control the input parameters and allows us to evaluate the performance of the SLODAR algorithm.

Blur is a flexible end-to-end solar AO simulation software that provides fast and accurate solar $\mathrm{AO}$ and $\mathrm{MCAO}$ simulations. It is written in $\mathrm{C}++$ and takes advantage of modern computer systems with a large number of cores to achieve fast performance. The code is heavily parallelized using OpenMP. The AO simulation code produces accurate WFS images by performing full physical simulations of the imaging process in a solar Shack-Hartmann WFS.

The simulation software has a modular design. It separates the AO physical simulation, performed by Blur, and the AO control, performed by KAOS. KAOS is a state of the art AO controller software designed by the Kiepenheuer-Institut für Sonnenphysik (KIS). KAOS is routinely used in modern solar telescopes to control their AO systems, such as in GREGOR and the pathfinder solar MCAO system Clear at the GST. Blur is the physical simulation part where the turbulent atmosphere and $\mathrm{AO}$ system components are simulated. At each iteration Blur generates solar WFS images that are sent to KAOS, which produces the WFS measurements through cross-correlations and updates the system's mirrors. The new mirror commands are sent back to Blur closing the loop. In this analysis we are only interested in open loop WFS measurements, thus, the control part of KAOS was not utilized. Only the WFS measurements produced by KAOS were stored and the AO control loop was never closed.

The WFS data used in this analysis was obtained by generating large numbers of random atmospheres, simulated as the combination of individual phase screens at different heights. Each phase screen was computed using the von Karman spectrum with a given value of Fried parameter (r0) and outer scale (L0). For each atmosphere, Blur generates a distorted WFS image and sends it to KAOS, which measures and stores the WFS shifts. ${ }^{4}$

\subsection{The wavefront sensor}

The WFS used in our SLODAR study is a wide field solar Shack-Hartmann WFS. The WFS divides the telescope pupil into square subapertures with a lenslet array. Each lenslet images a large fieldof-view image of the surface of the Sun producing an array of subaperture images in the camera 
of the WFS. Each subaperture image is subdivided into smaller sub-regions that correspond to different field directions in the sky. By cross-correlating these subregions independently for all the subapertures we obtain Shack-Hartman WFS measurements along different field directions.

We have matched the specifications of our simulated WFS to the WFS of Clear, the pathfinder solar MCAO system at the GST. Our goal is to understand the performance of SLODAR when it is applied to on-sky data captured by Clear. The WFS is composed by 112 subapertures arranged in a 12 x 12 grid. Each subaperture images 35" from which we select 7 sensing directions of 10 " size. This produces 112 pairs ( $\mathrm{x}$ and $\mathrm{y}$ ) of WFS shifts along 7 directions in the sky. ${ }^{5}$

\section{RESULTS}

\subsection{One-layer tests}

As a test of the algorithm, we started with the simplest case: a single layer. The first thing we noticed was that the performance improved dramatically as we increased the number of frames. This effect can be seen in Figure 4. The output in this case is a bar plot with each bar representing one turbulence layer in the reconstructed atmosphere. The results given generally have a correct height, but each layer has been split into multiple reconstructed layers, a problem that worsens when not enough frames are used. This example should be fairly straightforward to solve, as the reference functions and data were created under the same conditions, and no noise was added. We would expect that 10,000 fully independent frames would provide adequate statistical information for the algorithm to work perfectly; however, even with 40,000 frames, each layer is reconstructed as three.

For the next test, we experiment with increasing the height of the layers and compare how it works with using pinholes as objects and granulation. These results are shown in Figures 5 and 6 . The pinhole object is a point source, similar to what is used for nighttime astronomy, while solar granulation is what we would use in a real AO system for observing the sun. For our preliminary tests, we would like to know how it works in the nighttime case before moving on to study its performance with granulation. From this example, it appears the two cases work similarly, with the main difference being that the layer splitting problem is slightly more pronounced for granulation, especially at high altitudes.

\subsection{Three-layer tests}

After testing the performance of SLODAR for one turbulence layer, we test how well it reconstructs multiple layers. In Figure 7, we show the results for layers at 0,3 , and $6, \mathrm{~km}$; layers at 0,4 , and 8 $\mathrm{km}$; layers at 0,5 , and $10 \mathrm{~km}$; and layers at 0,6 , and $12 \mathrm{~km}$. Unsurprising, the algorithm performs similarly as it does for the one-layer case.

\subsection{Theoretical reference functions}

Up to this point, we have been constructing reference functions using a Monte Carlo simulation, where we generate thousands of random phase screens, and take the covariance of the resulting shifts. Figure 8 is the resulting profile for a single layer at $3 \mathrm{~km}$ when SLODAR is given reference covariance maps produced analytically, with $\mathrm{L} 0=100 \mathrm{~m}$, and the simulated data it is fitting is produced using $\mathrm{L} 0=5 \mathrm{~m}$. In Figure 9, the same reference functions are used, but the data is produced using $\mathrm{L} 0=100 \mathrm{~m}$. These results show that in this case, SLODAR performed much better when the L0 of the reference function is different from the L0 of the data used. This result does 

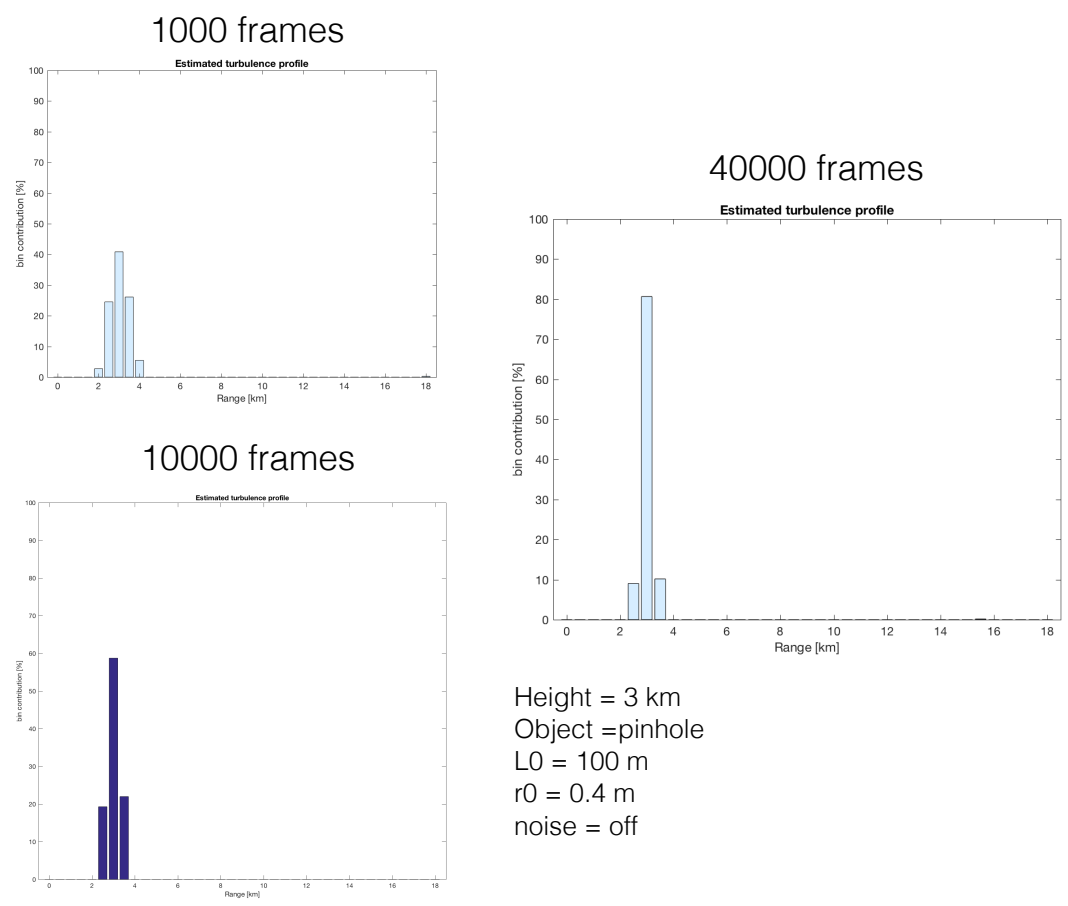

Height $=3 \mathrm{~km}$

Object $=$ pinhole

$\mathrm{LO}=100 \mathrm{~m}$

$\mathrm{rO}=0.4 \mathrm{~m}$

noise $=$ off

Figure 4. Turbulence profile given by SLODAR for a single turbulence layer at $3 \mathrm{~km}$, for 1000, 10000, and 40000 atmospheric phase screens. 

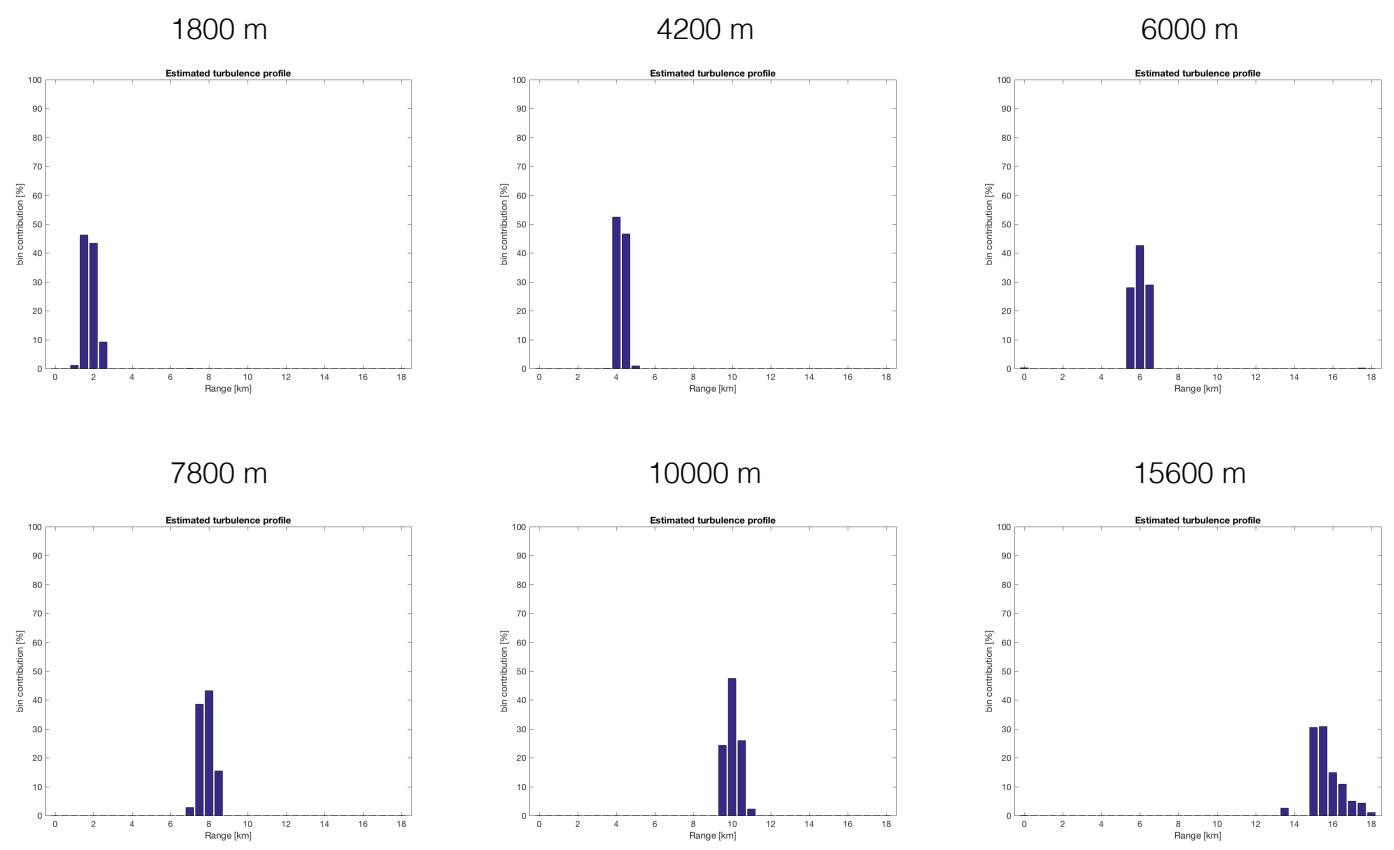

Figure 5. Layers reconstructed by SLODAR for single turbulence layers at various heights, using pinholes as target objects. 

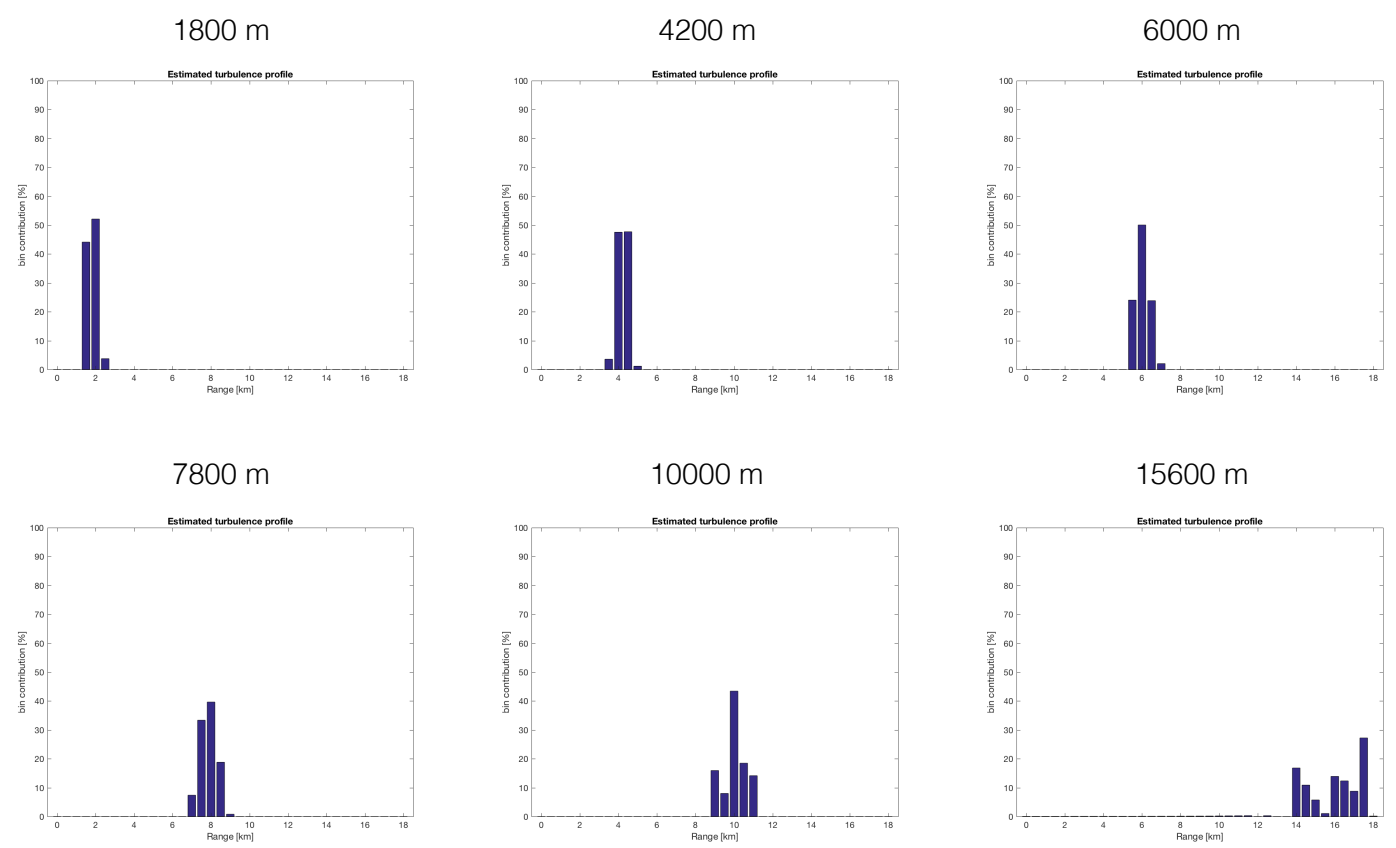

Figure 6. Layers reconstructed by SLODAR for single turbulence layers at various heights, using solar granulation as a target object. 

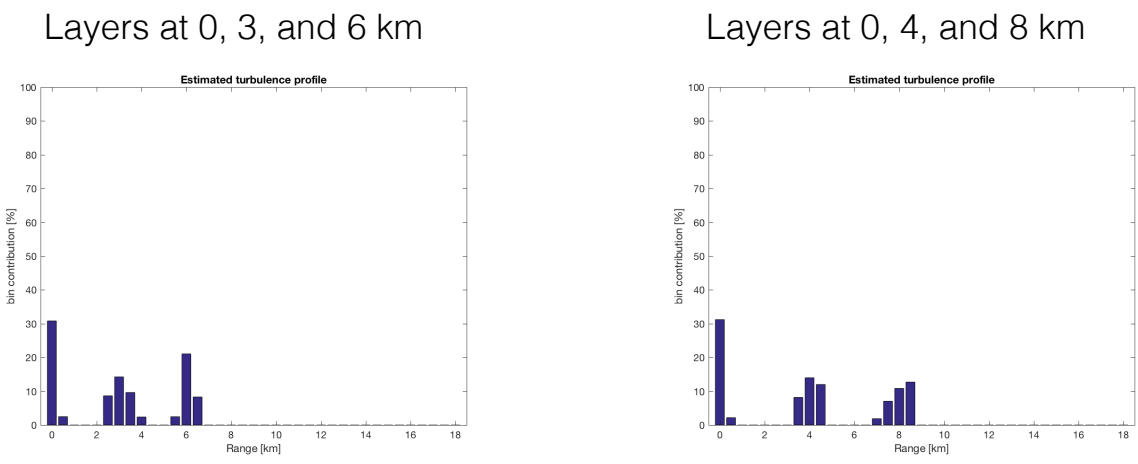

Layers at 0, 5, and $10 \mathrm{~km}$

Layers at 0, 6, and $12 \mathrm{~km}$
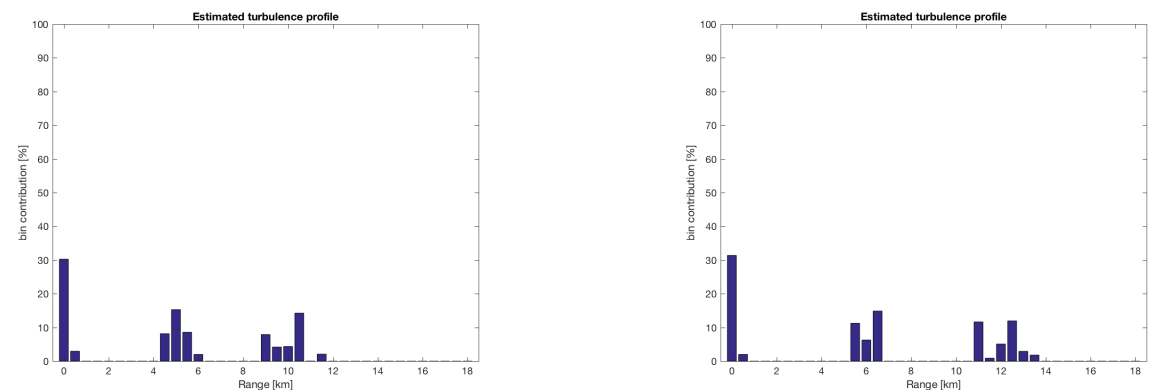

Figure 7. Layers reconstructed by SLODAR when testing with three turbulence layers at various heights, using solar granulation as a target object. 
not make sense, as we would expect that SLODAR would work best when reference functions and data are very similar. This shows that there is an inconsistency in our simulated data and may be evidence that the simulator used to produce data is not using the correct atmospheric statistics, and thus the L0 for the data is different from what we assume.

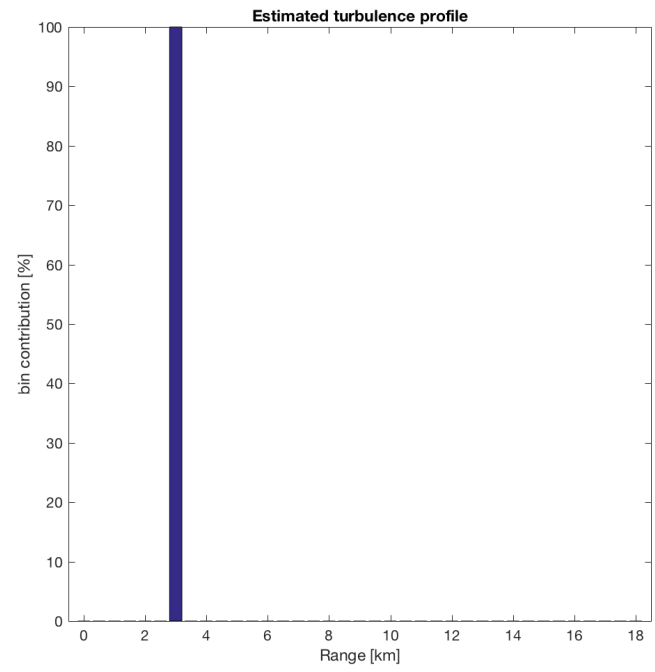

Figure 8. Profile given by SLODAR using reference covariance maps produced analytically, with L0 = 100 $\mathrm{m}$. For the input data, $\mathrm{L} 0=5 \mathrm{~m}$.

\section{ERROR ANALYSIS}

In most results SLODAR gives, when a single turbulence layer is fit to the reference functions, multiple layers are given as a solution. In perfect conditions (i.e. a single layer, no noise, atmospheric conditions matching the reference functions), we would expect the fit to work perfectly; however, this is not the case. It is not yet understood why this problem occurs.

In order to explore the error in the algorithm's given results, we can look at error maps, which are calculated by using the profile given by SLODAR to reconstruct a solution and then subtracting this answer from the covariance matrix or map given as input. An error map for a turbulence layer at $3 \mathrm{~km}$ is shown in Figure 10. On the left is the difference between the input matrix and the result given by the algorithm, and on the right is the difference between the input matrix and its corresponding reference function, which is what the error map would look like if the fit gave a perfect result. The main difference between these error maps is that in the one from the calculated solution, we can clearly see diagonal lines through some of the boxes, a remnant of the diagonal lines that form the peaks of the original covariance matrices. This means that the algorithm is not fitting perfectly to the strong signal of the peaks, but is instead matching the statistical noise.

\section{CONCLUSION}

We present preliminary results of a study to adapt the SLODAR technique to solar applications. We started by testing the algorithm for cases with one layer of turbulence in the atmosphere, 


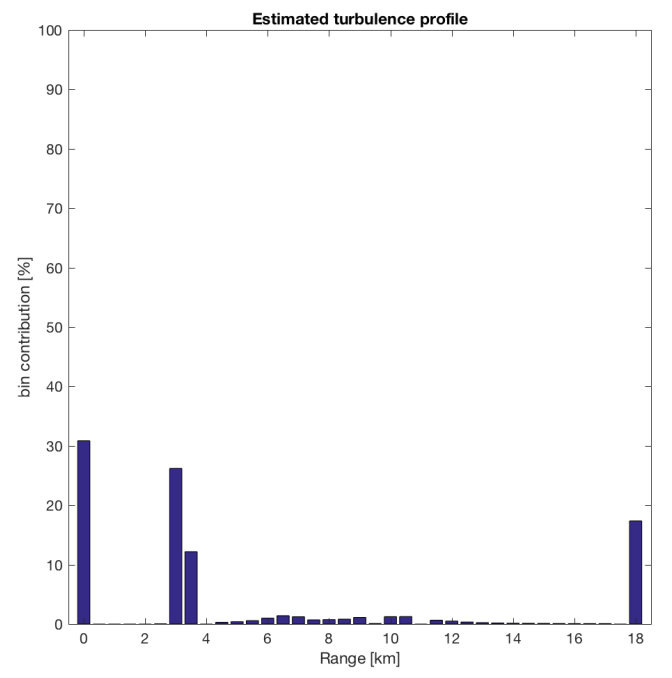

Figure 9. Profile given by SLODAR using reference covariance maps produced analytically. Both reference and input data use $\mathrm{L} 0=100 \mathrm{~m}$.

then with three layers. While it performed similarly when using granulation as target objects versus pinholes, we noticed that the main issue was that it would reconstruct each layer as several layers. This issue is most likely caused by statistical differences between the reference functions and the covariance matrices produced by test data. Using reference functions produced analytically gave promising results in reducing splitting, but strangely performed better when produced under different conditions than the data. This effect could potentially be caused by an error in the simulations and will have to be investigated further. In the future, we hope to provide a working implementation of SLODAR for Clear, the MCAO system at Big Bear Solar Observatory.

\section{REFERENCES}

1. Angela Cortés, Benoit Neichel, Andrés Guesalaga, James Osborn, Francois Rigaut, and Dani Guzman. Atmospheric turbulence profiling using multiple laser star wavefront sensors. Monthly Notices of the Royal Astronomical Society. 427, 2089-2099 (2012).

2. R. W. Wilson. SLODAR: measuring optical turbulence altitude with a Shack-Hartmann wavefront sensor. Monthly Notices of the Royal Astronomical Society. 337, 103-108 (2002).

3. T. Butterley, R. W. Wilson, and M. Sarazin. Determination of the profile of atmospheric optical turbulence strength from SLODAR data. Monthly Notices of the Royal Astronomical Society. 369, 835-845 (2006).

4. Jose Marino, Elizabeth Carlisle, and Dirk Schmidt. Simulation of DKIST solar adaptive optics system. Proc. SPIE 9909, Adaptive Optics Systems V, 99097C (2016).

5. Philip R. Goode and Wenda Cao. The 1.6 m off-axis New Solar Telescope (NST) in Big Bear. Proc. SPIE 8444, Ground-based and Airborne Telescopes IV, 844403 (2012). 

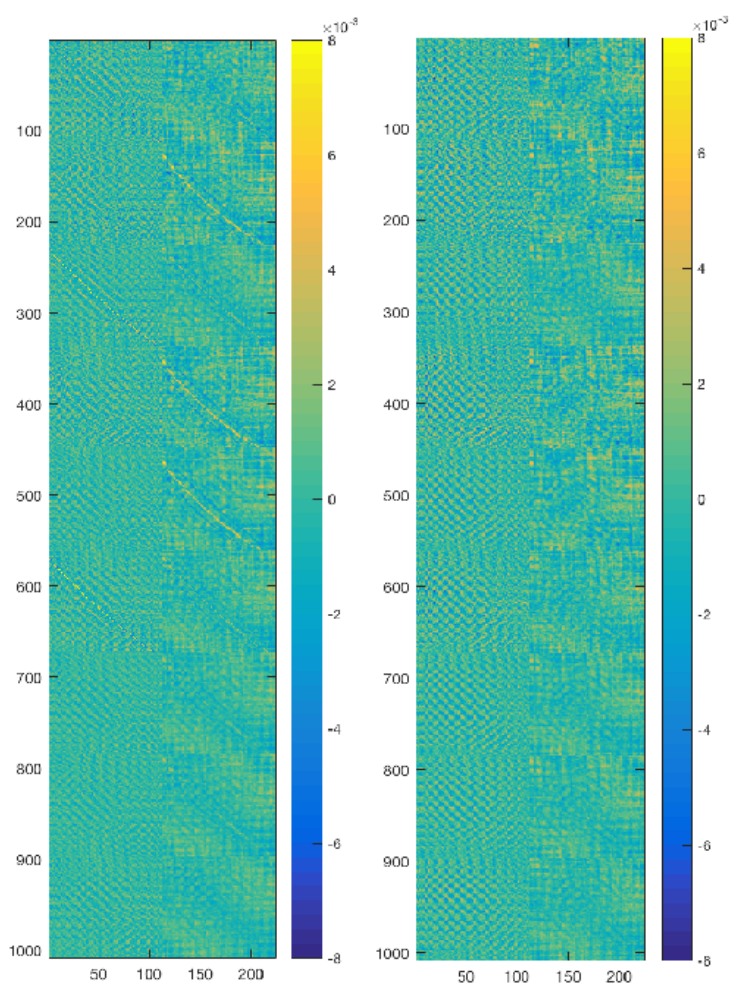

Figure 10. On the left is the difference between the solution given by SLODAR and the measured covariance matrix. On the right is the difference between the correct solution and the measured covariance matrix. 